\title{
Niños con mordeduras de animales hospitalizados en un centro de referencia de Uruguay
}

\author{
Martín Notejane*, Tatiana Moure, Jorge E. Da Silva, Patricia Barrios y Walter Pérez \\ Departamento de Pediatría, Facultad de Medicina, Universidad de la República, Hospital Pediátrico, Centro Hospitalario Pereira Rossell, Montevideo, \\ Uruguay
}

\section{Resumen}

Introducción: Las mordeduras de animales constituyen un problema sanitario. Pueden producir lesiones de diversa gravedad con riesgo de secuelas estéticas, funcionales y complicaciones infecciosas. El objetivo de este estudio fue describir las características clínicas y epidemiológicas, los tratamientos y las complicaciones de las lesiones por mordeduras de animales en niños hospitalizados en un centro de referencia de Uruguay. Métodos: Estudio descriptivo y retrospectivo. Se incluyeron todos los niños hospitalizados por mordeduras de animales entre 2014 y 2017. Se revisaron las historias clínicas y se registraron el animal involucrado, el tipo de lesiones, los tratamientos, las complicaciones y si el caso se notificó al Ministerio de Salud. Resultados: Se hospitalizaron 106 niños (media de edad: 4.5 años). El animal involucrado más común fue el perro (80.2\%), seguido de roedores (8.5\%), arácnidos (4.7\%) y otros (6.5\%). Las lesiones fueron en su mayoría únicas $(61.3 \%)$ y superficiales (80.2\%), y la hospitalización se hizo en cuidados moderados en el $91.5 \%$ de los casos. Los tratamientos se centraron en higiene (96.2\%), sutura (70.8\%) y profilaxis antibiótica (81.1\%). Se presentaron complicaciones en el $77.4 \%$ de los casos. En tres fue posible el aislamiento del agente etiológico más frecuente: Streptococcus pyogenes. El 20.7\% de los casos se notificaron al Ministerio de Salud. No se registró letalidad. Conclusiones: Del total de los niños hospitalizados, los más afectados fueron varones menores de 5 años. Predominaron las lesiones en la cabeza y los miembros, mayoritariamente leves y superficiales. En el 77.4\% de los casos hubo complicaciones, principalmente infecciosas y estético-funcionales.

Palabras clave: Mordeduras. Animales. Lesiones. Niños.

\section{Children with animal bites hospitalized in a reference center of Uruguay}

\section{Abstract}

Background: Animal bites are a health problem that can produce lesions of varying severity, with the risk of aesthetic and functional sequelae and infectious complications. The objective of the study was to describe the clinical, epidemiological, treatment and complications of lesions caused by animal bites in children hospitalized in a reference center in Uruguay. Method: Descriptive, retrospective study. All children hospitalized for animal bites between 2014 and 2017 were included. Medical records were reviewed and data, such as the animal involved, injuries, treatments, complications, and if the case was notified to the Ministry of Health, were recorded. Results: A total of 106 children were hospitalized, with a mean age

\section{Correspondencia:}

*Martín Notejane

E-mail: mnotejane@gmail.com
Fecha de recepción: 09-04-2018

Fecha de aceptación: 27-08-2018 DOI: 10.24875/BMHIM.18000031
Disponible en internet: 01-11-2018 Bol Med Hosp Infant Mex. 2018;75:358-365 www.bmhim.com

1665-1146/@ 2018. Hospital Infantil de México Federico Gómez, impreso por Permanyer México SA de CV, todos los derechos reservados. 
4.5 years. The animals involved were dogs (80.2\%), rodents (8.5\%), arachnids (4.7\%), and others $(6.5 \%)$. The majority of the lesions were superficial $(80.2 \%)$, and $91.5 \%$ of the cases were hospitalized in moderate care. The treatments were hygiene (96.2\%), suture (70.8\%), and antibiotic prophylaxis (81.1\%). There were complications in $77.4 \%$ of the cases. In three cases, the isolation of the most frequent etiological agent was possible: Streptococcus pyogenes. Notification to the Ministry of Health occurred in $20.7 \%$ of the cases. No lethality was recorded. Conclusions: A total of 106 children were hospitalized due to animal bites. The most affected were males under 5 years old. Most of them were bitten at home or nearby. Dogs and rodents were the most involved animals. There were predominantly head and limb lesions, mostly mild and superficial. Complications occurred in $77.4 \%$ of cases, mainly infectious and aesthetic-functional.

Key words: Bite. Animals. Injuries. Children.

\section{Introducción}

Las mordeduras de animales a niños constituyen un importante problema de salud ${ }^{1-13}$. Pueden producir lesiones de diversa gravedad, con riesgo de secuelas cicatriciales, funcionales, complicaciones infecciosas y daño psicoemocional, con elevados costos sanitarios ${ }^{1-8}$.

Las consecuencias para la salud humana dependen de la especie animal involucrada, del estado de salud del animal, de la edad y el estado de salud del niño víctima de la mordedura y de la capacidad de acceder a una atención sanitaria adecuada y oportuna ${ }^{4,7,9,10,12}$. Los niños pueden sufrir mordeduras de un gran número de especies animales; sin embargo, las más importantes en frecuencia son las causadas por perros y animales silvestres ${ }^{3-7,9}$.

Las mordeduras de perro son causa de decenas de millones de lesiones cada año, y los niños son quienes corren mayor riesgo. La rabia transmitida por mordeduras de perro y animales silvestres, como murciélagos y primates, constituye una gran preocupación para la salud ${ }^{3-11}$.

La hospitalización de niños por mordeduras de animales se debe mayoritariamente a sus complicaciones infecciosas y estético-funcionales, frecuentemente con la necesidad de reparación quirúrgica en bloque bajo anestesia ${ }^{3,6,7,13}$.

El presente estudio se llevó a cabo en el Hospital Pediátrico del Centro Hospitalario Pereira Rossell (HPCHPR), que es un hospital público de tercer nivel, centro de referencia nacional para la atención de niños y adolescentes, ubicado en Montevideo, Uruguay. Son escasos los estudios sobre este motivo de hospitalización. Describir y caracterizar este problema de salud podría contribuir a mejorar su abordaje.

El objetivo de este trabajo fue describir las características clínicas y epidemiológicas, los tratamientos y las complicaciones de las lesiones por mordeduras de animales en niños hospitalizados en un centro de referencia en Uruguay.

\section{Métodos}

Se realizó un estudio descriptivo, transversal y retrospectivo. Se incluyeron niños de 0 a 15 años hospitalizados por mordeduras de animales en el HP-CHPR, desde el primero de enero de 2014 hasta el 31 de diciembre de 2017. Se realizó la revisión de las historias clínicas en formato de papel.

Se analizaron las siguientes variables: edad, sexo, circunstancia de la mordida (mes del año y lugar) y animal involucrado. En caso de mordedura por can, se indagaron el nombre del propietario, el estado de salud del animal, la vacuna antirrábica, los síntomas de rabia y la muerte del animal.

Con respecto a las lesiones observadas en el examen clínico, se indagaron el número, la topografía, la profundidad, el tipo, la gravedad y los tratamientos. Se analizó la presencia del registro de notificación del evento a la Unidad de Zoonosis y Vectores, División de Epidemiología del Ministerio de Salud, y al Centro de Información y Asesoramiento Toxicológico, de la Cátedra de Toxicología, Facultad de Medicina.

Se registraron el lugar de hospitalización (sala de cuidados moderados o cuidados intensivos), los días de hospitalización y las complicaciones.

Para los efectos de este estudio, se consideró lesión por mordedura grave aquella que, directamente o por cercanía con vasos sanguíneos o la vía aérea, implicara riesgo vital real o potencial, comprometiera planos profundos (fascia, músculo, periostio), presentara lesiones desgarrantes extensas con pérdida de sustancia 0 provocara pérdida parcial o total de alguna función. También se consideró como lesión grave o potencialmente grave todo evento por mordedura de animal ponzoñoso (arácnidos, ofidios).

\section{Análisis estadístico}

Las variables cualitativas se expresaron como frecuencias absolutas y relativas (\%); las variables 


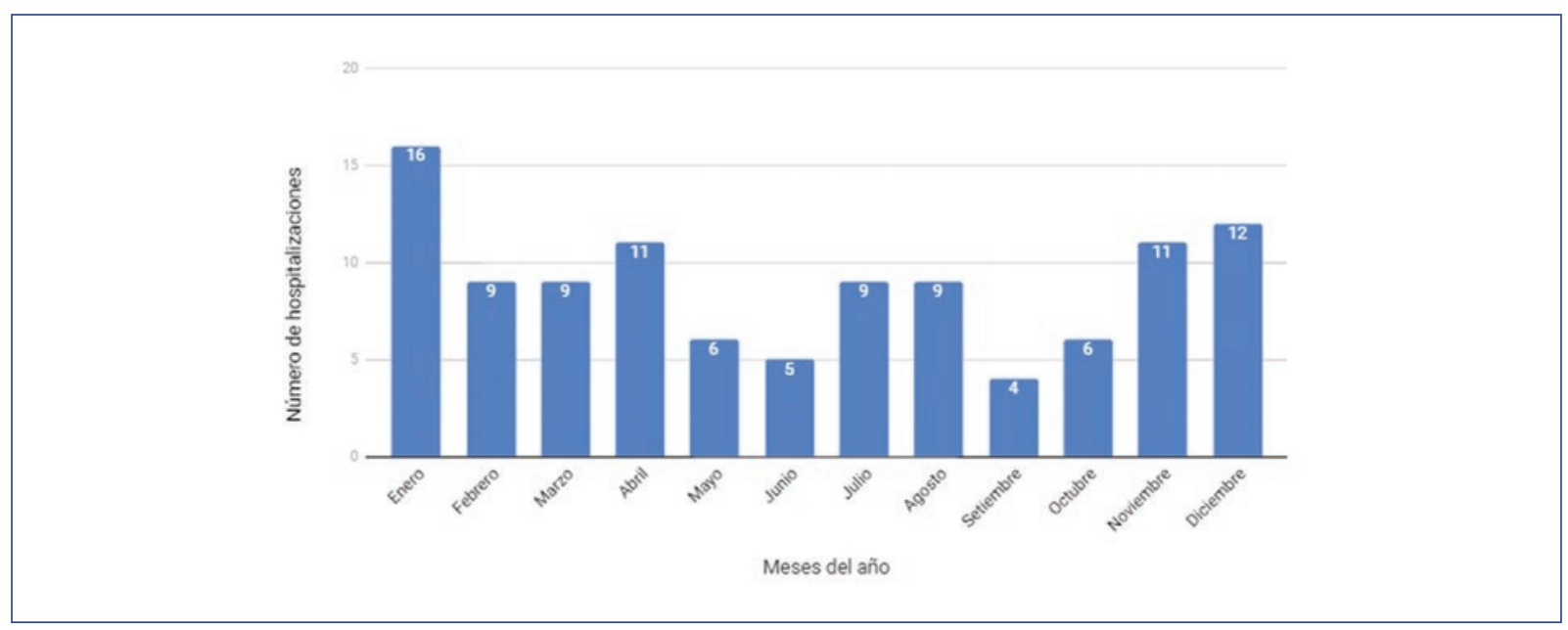

Figura 1. Distribución mensual de las hospitalizaciones por mordeduras de animales en un centro de referencia en Uruguay $(n=106)$.

cuantitativas, en medidas de tendencia central y rango. El análisis de las variables dicotómicas y categóricas se realizó con las pruebas $\chi^{2}$, de Pearson o exacta de Fisher, según correspondiera, asumiendo un nivel de significación estadística de $p<0.05$. Para procesar los datos se utilizó el programa Epi Info' ${ }^{\mathrm{TM}}$, versión 7.2.

Se contó con la autorización de la Dirección del Hospital Pediátrico y la aprobación por el Comité de Ética de Investigación del centro para la realización de este estudio.

\section{Resultados}

Durante el periodo de estudio fueron hospitalizados 106 niños por mordeduras de animales, correspondiendo al 9.2\% (106/1152) de las consultas por este motivo en el Departamento de Emergencia Pediátrica del HP-CHPR ${ }^{a}$.

La media de edad al ingreso fue de 4.5 años (4 meses a 15 años). El $62.3 \%(n=66)$ fueron menores de 5 años, y el $57.5 \%(n=61)$ eran de sexo masculino. La consulta fue inmediata a la mordida en el $76.4 \%$ $(n=81)$. En el $23.6 \%(n=25)$, la consulta se realizó en la evolución, motivada por alguna complicación.

Las lesiones por mordeduras predominaron en los meses de primavera y verano $(54.7 \% ; n=58)$ y el $67.9 \%(n=72)$ se produjeron en el domicilio o sus alrededores. En la figura 1 se expresa la distribución por

a Datos proporcionados por el Departamento de Registros Médicos del HP-CHPR. mes de la hospitalización por mordeduras de animales en la población incluida.

Los animales involucrados, en primer lugar, fueron perros $(80.2 \%, n=85)$, seguidos de roedores $(8.5 \%$, $n=9)$ y arácnidos $(4.7 \%, n=5)$. En la tabla 1 se muestran las especies involucradas. En cuanto a las mordeduras de perro, en el $41.2 \%$ de los casos el perro era conocido por el niño (vecino, familiar), en el $22.4 \%$ era propio, en el $18.6 \%$ era desconocido y en el $40.2 \%$ no se contaba con el dato. La observación del can por 10 días se registró únicamente en el $11.8 \%$ de los casos. Se especificó que no existía clínica sugestiva de rabia en el $13 \%$. Se registró vacunación antirrábica en el $14.2 \%$; el $7.1 \%$ de los perros la presentaba vigente. No se constataron registros de fallecimientos de canes. Se encontró un importante subregistro de las variables vinculadas al estado de salud y a la inmunidad del perro agresor.

En cuanto a las lesiones por mordeduras de animales de los niños hospitalizados, se encontraron como únicas el $61.3 \%(n=65)$, superficiales el $80.2 \%(n=85)$ y en su mayoría de tipo laceración $(60.4 \%, n=64)$. La topografía más frecuente fue la cara $(51.8 \%)$, seguida por los miembros superiores (31.1\%). En los menores de 5 años predominaron las lesiones en la cabeza y el cuello $(56 \%, n=37 ; p<0.045)$; en los mayores de 5 años se presentaron lesiones de miembros $(47.5 \%$, $n=19 ; p=0.076$ ).

Se detectaron lesiones leves en el $67.9 \%(n=72)$ y graves en el $32.1 \%(n=34)$. Dentro de las graves, 24 de las 34 fueron por canes $(p<0.031)$, y 5 de los 24 perros eran de raza pitbull. En la tabla 2 se muestran 
Tabla 1. Especies de animales involucrados en las mordeduras a niños hospitalizados en un centro de referencia en Uruguay $(n=106)$

\begin{tabular}{|l|c|c|}
\hline Especie animal & $\begin{array}{c}\text { Frecuencia } \\
\text { absoluta (n) }\end{array}$ & $\begin{array}{c}\text { Frecuencia } \\
\text { relativa (\%) }\end{array}$ \\
\hline Perro/can & 85 & 80.2 \\
$\quad$ No se identificó la raza & 56 & 65.8 \\
Pitbull & 20 & 23.5 \\
Cimarrón & 4 & 4.7 \\
Rottweiler & 2 & 2.3 \\
Ovejero alemán & 1 & 1.1 \\
Fila & 1 & 1.1 \\
\hline Cruza/mestizo & 1 & 1.1 \\
\hline Roedor & 9 & 8.5 \\
\hline Arácnido & 5 & 2.8 \\
\hline Loxosceles laeta & 3 & \\
\hline No se identificó la especie & 2 & 2.8 \\
\hline Ofidio & 3 & \\
\hline Bothrops alternatus (crucera) & 2 & 1.8 \\
\hline No se identificó la especie & 1 & 0.9 \\
\hline Murciélago & 2 & 0.9 \\
\hline Equino & 1 & \\
\hline Porcino & 1 & \\
\hline
\end{tabular}

las características clínicas de las lesiones producidas por las mordeduras de los animales.

Se identificaron complicaciones en el $77.4 \%(n=82)$ de los niños: el $47.6 \%$ de tipo infecciosas $(n=39)$, celulitis en 30 de 39, y abscesos en 7 de 39 . El 39\% de las complicaciones fueron estético-funcionales $(n=32)$ : cicatrices (25/32), leves (18/25), moderadas o graves con necesidad de injertos o amputación (7/25: tres niños con secuelas graves de cara, tres amputaciones de falanges, un arrancamiento completo de pabellón auricular) o funcionales (7/32: cinco niños con hemiparesia/hemiplejia, dos parálisis facial por lesión del séptimo par craneano). Se registró un caso de loxoscelismo cutáneo-visceral o sistémico en un paciente de sexo masculino de 5 años que acudió a consulta por mal estado general y palidez ictérica, agregando en la evolución hematuria microscópica en apirexia. En la tabla 3 se expresan las complicaciones registradas.

Se solicitaron estudios microbiológicos en el $21.7 \%$ ( $n=23)$ : cultivos de la lesión $(15 / 23)$ y hemocultivos (10/23). Se aisló el agente etiológico en tres casos: en dos Streptococcus pyogenes, y en uno, Pasteurella canis.

Se indicó profilaxis con antibióticos en el $81.1 \%(n=86)$. El antibiótico más prescrito fue ampicilina-sulbactam intravenoso, en el $58.1 \%(n=50)$, con una media de duración del tratamiento de 8 días (rango: 1 a 36 días).
Tabla 2. Características clínicas de las lesiones producidas por mordeduras de animales en niños hospitalizados en un centro de referencia en Uruguay $(n=106)$

\begin{tabular}{|l|c|c|}
\hline Variables & $\begin{array}{c}\text { Frecuencia } \\
\text { absoluta (n) }\end{array}$ & $\begin{array}{c}\text { Frecuencia } \\
\text { relativa (\%) }\end{array}$ \\
\hline $\begin{array}{l}\text { Número } \\
\text { Únicas } \\
\text { Múltiples }\end{array}$ & 65 & 61.3 \\
\hline $\begin{array}{l}\text { Profundidad } \\
\text { Superficiales }\end{array}$ & 41 & 39 \\
\hline Profundas & & \\
\hline Tipo & 85 & 80.2 \\
\hline Laceración & 21 & 19.8 \\
\hline Punzante/penetrante & & \\
\hline Abrasión & 64 & 60.4 \\
Arañazo & 24 & 22.6 \\
Sin dato & 2 & 1.9 \\
\hline Localización & 14 & 1.9 \\
Cabeza- cuello & & 13.2 \\
\hline Miembros & 55 & \\
Múltiples topografías & 42 & 51.9 \\
\hline Tronco & 7 & 39.6 \\
\hline Gravedad & 2 & 6.6 \\
Leves & & 1.9 \\
\hline Graves & & \\
\hline
\end{tabular}

Al momento de la hospitalización, el 78.3\% ( $n=83)$ de los niños presentaba vacuna antitetánica vigente. Se administraron ocho refuerzos de vacuna antitetánica, se iniciaron tres esquemas con vacuna antirrábica y gammaglobulina (dos por mordedura de murciélago y uno por perro que se utilizaba para la caza $)^{b}$. Se utilizaron antídotos en cuatro pacientes (tres por mordedura de ofidio y uno por arácnido) ${ }^{c}$. En los casos de mordedura por ofidio, en dos de ellos se identificaron ejemplares de la especie Bothrops alternatus; en el caso restante no se logró identificar la especie, pero provenía de una zona de presencia frecuente de esta especie. En el caso del arácnido, el antídoto se administró a un paciente de 3 años que acudió a consulta a las 48 horas posteriores a la mordedura, con loxoscelismo visceral; no se registraron otros tratamientos. En la tabla 4 se muestran los tratamientos realizados. Se encontró el registro de la notificación del evento (mordedura) en el $20.7 \%$ de los casos $^{2,3}$.

\footnotetext{
b Datos proporcionados por la Unidad de Zoonosis y Vectores, División Epidemiología, Ministerio de Salud.

c Datos proporcionados por el Centro de Información y Asesoramiento Toxicológico, Cátedra de Toxicología, Facultad de Medicina, Universidad de la República.
} 
Tabla 3. Complicaciones de las mordeduras por animales en los niños hospitalizados en un centro de referencia en Uruguay $(n=82)$

\begin{tabular}{|c|c|c|}
\hline Complicaciones & $\begin{array}{l}\text { Frecuencia } \\
\text { absoluta (n) }\end{array}$ & $\begin{array}{l}\text { Frecuencia } \\
\text { relativa (\%) }\end{array}$ \\
\hline $\begin{array}{l}\text { Infecciosas } \\
\text { Celulitis } \\
\text { Abscesos } \\
\text { Fiebre por mordedura de roedor }\end{array}$ & $\begin{array}{c}39 \\
30 \\
7 \\
2\end{array}$ & 47.6 \\
\hline $\begin{array}{l}\text { Estético/funcionales }^{*} \\
\text { Cicatrices } \\
\text { Leves } \\
\text { Moderadas-graves } \\
\text { Funcionales } \\
\text { Hemiparesia/hemiplejia } \\
\text { Parálisis facial }\end{array}$ & $\begin{array}{l}32 \\
25 \\
18 \\
7 \\
7 \\
5 \\
2\end{array}$ & 39 \\
\hline $\begin{array}{l}\text { Ortopédicas/traumatológicas* } \\
\text { Fracturas } \\
\text { Cráneo-cara } \\
\text { Miembro superior (radio) } \\
\text { Traumatismo encefalocraneano } \\
\text { Leve } \\
\text { Grave con secuela neurológica }\end{array}$ & $\begin{array}{l}11 \\
8 \\
7 \\
1 \\
6 \\
4 \\
2\end{array}$ & 13.4 \\
\hline $\begin{array}{l}\text { Hemorrágicas/hematológicas } \\
\text { Hemorragia moderada/shock } \\
\text { hipovolémico } \\
\text { Trastorno de coagulación }\end{array}$ & $\begin{array}{l}11 \\
8 \\
3\end{array}$ & 13.4 \\
\hline Dolor & 1 & 1.2 \\
\hline $\begin{array}{l}\text { Viscerales } \\
\text { Loxoscelismo visceral o } \\
\text { sistémico. }\end{array}$ & $\begin{array}{l}1 \\
1\end{array}$ & 1.2 \\
\hline
\end{tabular}

*Se encontró registro simultáneo de diferentes complicaciones estético-funcionales y ortopédicas.

La hospitalización inicial se realizó en salas de cuidados moderados en el 91.5\% $(n=97)$ de los casos, requiriendo el ingreso a la unidad de cuidados intensivos el $8.5 \%(n=9)$ : siete por mordedura de can y dos por mordedura de ofidio. El principal motivo de ingreso en la unidad de cuidados intensivos fueron las complicaciones traumatológicas de cara y cráneo (6/9). La media total de días de hospitalización fue de 4.25 días (rango: 1 a 42 días).

Del total de los niños hospitalizados por mordeduras en el periodo de estudio, dos reingresaron en la evolución: uno debido complicaciones infecciosas y el otro para una nueva reparación quirúrgica. No se registró letalidad durante el periodo.

\section{Discusión}

Se estima que el $0.5-1 \%$ de las consultas en urgencias pediátricas se deben a mordeduras de animales ${ }^{1,2,13}$, y que
Tabla 4. Tratamientos aplicados a los niños hospitalizados por mordeduras de animales $(n=106)$

\begin{tabular}{|l|c|c|}
\hline Variables & $\begin{array}{c}\text { Frecuencia } \\
\text { absoluta (n) }\end{array}$ & $\begin{array}{c}\text { Frecuencia } \\
\text { relativa (\%) }\end{array}$ \\
\hline Higiene & 102 & 96.2 \\
\hline Suturas & 75 & 70.7 \\
\hline Antibiótico profiláctico & 86 & 81.1 \\
\hline $\begin{array}{l}\text { Ampicilina-sulbactam solo o } \\
\text { asociado }\end{array}$ & 50 & 58.1 \\
\hline $\begin{array}{l}\text { Amoxicilina-ácido clavulánico } \\
\text { solo o asociado }\end{array}$ & 27 & 31.4 \\
\hline Cefradina & 4 & 4.6 \\
\hline Clindamicina sola o asociada & 2 & 2.3 \\
\hline Penicilina cristalina & 1 & 1.2 \\
\hline Cefuroxima axetilo & 1 & 1.2 \\
\hline Trimetoprima-sulfametoxazol & 1 & 1.2 \\
\hline Sin dato & 5 & 4.7 \\
\hline No corresponde & 15 & 14.1 \\
\hline
\end{tabular}

se hospitalizan el $10-30 \%$ de los niños que consultan por este motivo, en concordancia con lo registrado en esta serie $^{1,3,5-7,13}$.

Se presenta una revisión de 4 años de hospitalizaciones por mordeduras de animales en un centro de referencia pediátrico del subsector público del Uruguay. Es necesario puntualizar que este problema de salud, según refiere la bibliografía, se encuentra relacionado con la pobreza y las malas condiciones del control de la fauna canina ${ }^{3,8,11}$, por lo que los resultados de este estudio no se pueden generalizar a otros centros de Uruguay y de la región.

Los varones en edad escolar fueron el grupo más afectado; datos similares han sido reportados por la literatura nacional e internacional ${ }^{1-6}$. Los eventos predominaron durante los meses de primavera y verano, fueron domiciliarios o peridomiciliarios, siendo más frecuentes en horas vespertinas, los fines de semana, en días festivos y en los meses de calor, vinculados con la recreación infantil| ${ }^{3-5,8}$.

Los perros fueron los animales más frecuentemente involucrados, representando un importante problema de salud-11,13; esto puede deberse a que son las mascotas preferidas por las familias. De acuerdo con una encuesta telefónica realizada en mayo de 2017 en Uruguay por el Ministerio de Ganadería, Agricultura y Pesca, el $67 \%$ de los hogares tenían al menos una 
mascota, en su mayoría perros. Uruguay presenta una población canina actual de 1,740,000 perros, sin contar a los perros sin dueño. Esta cifra permite afirmar que existen tres perros por cada 10 habitantes, superando las recomendaciones de la Organización Mundial de la Salud, de un perro por cada 10 habitantes ${ }^{d}$.

La bibliografía refiere la existencia de razas de perros más "agresivos» (pitbull, cimarrón, rottweiler y ovejero alemán), las cuales presentan alto riesgo y han sido implicadas en episodios graves de mordeduras en niños ${ }^{1-3,6,9}$. El dato de la raza carece de valor si se desconoce la proporción que ocupa cada raza en el total de la población canina de un país. Además, es conocido que la tendencia a morder de un perro depende de otros factores distintos de la raza: herencia, socialización, entrenamiento, experiencias previas, estado de salud del animal y conducta de la víctima ${ }^{3,9}$. En esta serie, las razas más identificadas fueron el pitbull y a continuación el cimarrón, coincidiendo con los datos reportados por Cedrés, et al. ${ }^{13}$ Cabe destacar que estas razas no son las predominantes en el país, según datos del Ministerio de Ganadería, Agricultura y Pesca, siendo más frecuente en los hogares el perro mestizo o cruza $(47 \%)$, seguido del caniche $(11 \%)$ y el pastor alemán $(6 \%)^{3}$. En su mayoría, la mordedura la realizó un perro conocido, que puede ser propio o del vecino, lo cual es coincidente con los datos reportados ${ }^{1-3,6,9,13}$.

Los roedores fueron la segunda especie animal en frecuencia causante de hospitalización. Se observa en todo el mundo un incremento de lesiones y mordeduras por roedores en áreas urbanas y rurales ${ }^{7,9,10}$, así como un incremento en los reportes de casos de enfermedades transmitidas por estas especies. Los niños que sufren mordeduras de ratas son provenientes de zonas vulnerables, de bajo nivel socioeconómico y cultural, con escaso saneamiento y dificultades de acceso al agua potable ${ }^{10}$.

Las mordeduras de animales en esta serie produjeron lesiones, en su mayoría únicas y superficiales, de carácter leve. La cabeza y el cuello fueron los sectores más comprometidos, seguidos por los miembros superiores. La afectación de la cabeza y el cuello fue más frecuente en niños menores de 5 años, encontrándose una asociación estadísticamente significativa ${ }^{1-6,8}$. Un dato discordante en los diversos estudios previos es el análisis de la gravedad de las lesiones producidas por mordeduras de animales. Esta variabilidad puede deberse a las

d Comisión Nacional Honoraria de Tenencia Responsable y Bienestar Animal, Ministerio de Agricultura Ganadería y Pesca, Encuesta de Equipos Consultores, mayo 2017. distintas definiciones adoptadas para considerar como grave o potencialmente grave una mordedura.

El porcentaje de complicaciones por mordedura de animal es heterogéneo (10-50\%), dependiendo de la edad del niño, del estado de salud e inmunidad, de la topografía de la lesión y su profundidad, y del animal involucrado ${ }^{5,6,13,14}$. Las complicaciones y la necesidad de cirugía reparadora bajo anestesia en bloque quirúrgico constituyen el principal motivo de hospitalización en la población pediátrica, como se observó en esta serie. Predominaron las complicaciones infecciosas, seguidas de las estéticas, a diferencia de lo reportado previamente en trabajos nacionales ${ }^{13}$.

Entre las complicaciones infecciosas, las más frecuentes fueron celulitis y abscesos, refiriéndose también en la literatura la tenosinovitis, la artritis séptica, la osteomielitis y la septicemia, entre otras ${ }^{3,5,6}$. La mayoría de las infecciones vinculadas a mordeduras por animales son de etiología polimicrobiana, siendo los agentes aerobios aislados con mayor frecuencia Staphylococcus aureus, Staphylococcus epidermidis, Streptococcus sp. y Pasteurella multocida, y predominando entre los anaerobios Fusobacterium sp. y Bacteroides ${ }^{1,4,8,12,14-18}$.

Existen controversias en las recomendaciones que justifican la prescripción y la duración de los tratamientos antimicrobianos ${ }^{1,4}$. La recomendación de prescribir antibióticos profilácticos está dada por la presencia de factores de riesgo para la infección, la presentación clínica y la existencia de parámetros infecciosos ${ }^{1,8}$. Se describen como factores de riesgo para infección la edad (menor de 1 año), las heridas punzantes localizadas en manos, pies, cara y cuello, las heridas próximas o contiguas a vasos sanguíneos, linfáticos 0 articulación protésica, así como las heridas por mordedura de gato, las que motivaron consulta tardía (más de 12 horas) o se producen en un huésped inmunodeprimi$\mathrm{do}^{1,4,5,8,9,12,14}$. El antibiótico recomendado en la mayoría de las guías internacionales es amoxicilina-ácido clavulánico por 3 a 5 días, de acuerdo con la evolución. Este antimicrobiano es efectivo dada su acción sobre las bacterias aerobias y anaerobias de la cavidad oral de la inmensa mayoría de los animales y la flora propia de la piel del paciente ${ }^{1,4,8,9,12,14}$. Se registraron dos casos de fiebre probable por mordedura de rata, siendo una situación que debe tenerse en cuenta por su potencial gravedad en todo paciente que haya sufrido una mordedura por un roedor y presente fiebre en la evolución 7,10 .

La inmensa mayoría de los niños hospitalizados requirieron sutura. La bibliografía refiere que la mayor 
parte de las heridas por mordeduras pueden ser suturadas si la consulta es precoz y si se aplica una buena técnica quirúrgica, asociándose con una menor posibilidad de infección respecto a las que se mantienen abiertas $^{1,19-22}$. En general, las heridas pequeñas y simples en los miembros pueden permanecer abiertas bajo vigilancia; en cambio, las heridas extensas localizadas en la cara y el cuello deben ser suturadas y recibir estricto control ${ }^{1}$. Los expertos recomiendan enfáticamente que la reparación se haga en el menor tiempo posible, dado que esto se asocia con una cicatrización menos hipertrófica y con menor posibilidad de secuelas cicatriciales y funcionales. Además, en caso de existir lesiones subyacentes a nervios, tendones 0 vasos sanguíneos, estos pueden repararse desde un inicio, lo que repercute en una mejor recuperación funcional posterior con menor número de secuelas estéticas $^{19-22}$. En esta serie, las complicaciones estético-funcionales fueron las segundas en frecuencia. $\mathrm{Si}$ bien las cicatrices leves fueron las más registradas, se encontró un número no despreciable de cicatrices moderadas-graves con secuelas funcionales por lesión de nervios. La literatura refiere que la cara y los miembros son los sectores más afectados por las secuelas cicatriciales, dejando en muchos casos cicatrices queloides poco estéticas, y en ocasiones con afectación de la funcionalidad, principalmente cuando se localizan en las articulaciones de las manos o los pies ${ }^{20,21}$. Las lesiones de nervios y sus secuelas, como se registró en este estudio, requieren un abordaje quirúrgico particular, como puede ser la utilización de colgajos de piel e injertos cutáneos que pueden utilizarse de manera conjunta o individual ${ }^{22}$. Una adecuada evaluación inicial analizando la gravedad de la herida con el uso de escalas de gravedad, como la descrita por Lackmann, permitirá individualizar el tratamiento adecuado en cada caso ${ }^{21,22}$.

Las complicaciones psicoemocionales no fueron evaluadas en el presente estudio. La bibliografía describe casos de estrés postraumático, pesadillas y temor a los animales ${ }^{2,5}$, por lo cual es importante considerar el abordaje psicoemocional desde el inicio de la intervención.

En consideración a los tratamientos específicos, se evidenció una baja incidencia de profilaxis antirrábica, probablemente vinculada a las normativas del Ministerio de Salud, que realiza el seguimiento de la enfermedad. Uruguay es considerado un país libre de rabia humana desde 1966, y el último caso de rabia canina se registró en $1983^{1,23}$. De los tres casos en que se indicó el esquema de vacuna más inmunoglobulina antirrábica, dos eran niños mordidos por murciélagos, animales al norte del país que se han reportado como transmisores de rabia a otros animales (vacas, caballos y ovejas), y el otro fue por mordedura de perro de caza, siendo este un factor de riesgo para rabia descrito en la literatura ${ }^{23}$.

La indicación de sueros antiarácnidos (antiloxosceles) es discutida, ya que su plazo de indicación más efectivo es durante las primeras $36-72$ horas tras la mordedura, así como en formas sistémicas en presencia de hemólisis, como en el caso que presentó loxoscelismo cutáneo-visceral ${ }^{24,25}$. Se trata de un cuadro clínico de mayor gravedad y se presenta en el $16 \%$ de los casos en adultos, con una mortalidad descrita del $1-3 \%{ }^{25}$. Ocurre cuando el veneno alcanza la circulación sistémica, tanto por inoculación directa en un capilar como por alteración de la permeabilidad, ejerciendo un gran poder hemolítico. Se caracteriza por una gran afectación del estado general acompañada de ictericia, palidez, hematuria, hemoglobinuria y fiebre durante las primeras 12-24 horas posteriores a la mordedura. La hemoglobinuria y la hematuria están presentes en el $100 \%$ de los casos. La gravedad de la manifestación cutánea no predice su evolución ${ }^{25}$; en cambio, la indicación de suero antiofídico ante la sospecha de mordedura por especie Bothrops debe realizarse en forma urgente, previa administración de corticoides, para minimizar la posibilidad de anafilaxia ${ }^{25-29}$.

La principal limitación de este estudio fue el tipo de diseño metodológico seleccionado. Al tratarse de un estudio retrospectivo, se detectaron problemas en la calidad de los registros médicos al revisar las historias clínicas. Se destaca que en un importante número de historias no se encontraba el dato del propietario del animal involucrado, la raza del perro, el estado inmunitario del animal, la posibilidad de observación clínica de este por 10 días, los síntomas sugestivos de rabia animal ni la notificación obligatoria del evento de la mordedura animal. La realización de estudios de tipo prospectivo podría minimizar esta brecha. Si bien los autores acordaron una definición operativa de lesiones por mordedura grave, el uso de escalas de gravedad de las heridas, como la de Lackmann, permitiría realizar un análisis más profundo de las complicaciones estético-funcionales. Como fortaleza, hasta nuestro conocimiento, este es el primer estudio nacional que describe este motivo de hospitalización y sus complicaciones.

Como conclusiones podemos enunciar que en los últimos 4 años se hospitalizaron 106 niños por mordeduras de animales en el centro de referencia público de Uruguay, siendo los más afectados varones 
menores de 5 años, mordidos en el domicilio o en sus proximidades. Los perros y los roedores fueron los animales más involucrados. Predominaron las lesiones en la cabeza y los miembros, mayoritariamente únicas, superficiales, de tipo laceración y de carácter leve. Se registraron complicaciones en el $76 \%$ de los niños hospitalizados por este motivo, siendo las más frecuentes las complicaciones infecciosas, seguidas de las estético-funcionales.

\section{Responsabilidades éticas}

Protección de personas y animales. Los autores declaran que para esta investigación no se han realizado experimentos en seres humanos ni en animales.

Confidencialidad de los datos. Los autores declaran que han seguido los protocolos de su centro de trabajo sobre la publicación de datos de pacientes.

Derecho a la privacidad y consentimiento informado. Los autores han obtenido el consentimiento informado de los pacientes $y / 0$ sujetos referidos en el artículo. Este documento obra en poder del autor de correspondencia.

\section{Conflicto de intereses}

Los autores declaran no tener ningún conflicto de intereses

\section{Financiamiento}

No se contó con financiamiento para esta investigación.

\section{Bibliografía}

1. Glausiuss G, Ascione A, Sehabiague G. Mordeduras por animales en la edad pediátrica. Arch Pediatr Urug. 2000;71:24-30.

2. Blanco M, Pérez W. Mordeduras de perro en niños. Arch Pediatr Urug. 2004:75:120-4

3. Schvartzman S, Pacín M. Lesiones por mordedura de perro en niños. Arch Arg Pediatr. 2005;103:389-95.

4. Morgan M. Hospital management of animal and human bites. J Hosp Infect. 2005;61:1-10.

5. Schalamon J, Ainoedhofer H, Singer G, Petnehazy T, Mayr J, Kiss K, et al. Analysis of dog bites in children who are younger than 17 years. Pediatrics. 2006;117:e374-9.
6. Morales $\mathrm{C}$, Falcón $\mathrm{N}$, Hernández $\mathrm{H}$, Fernández $\mathrm{C}$. Accidentes por mordedura canina, casos registrados en un hospital de niños de Lima, Perú 1995-2009. Rev Peru Med Exp Salud Pública. 2011;28:639-42.

7. Carbejos G, Hernández H, Fernández C, Falcón N. Accidentes causados por animales silvestres, sinantrópicos y de producción en un hospital de niños en Lima, Perú: estudio retrospectivo periodo 1995- 2009. Una Salud. Rev Sapuvet de Salud Pública. 2011;2:15-27.

8. Ellis R, Ellis C. Dog and cat bite. Am Fam Physician. 2014;90:239-43.

9. Samanta M, Mondal R, Shah A, Hazra A, Ray S, Dhar G. Animal bites and rabies prophylaxis in rural children: Indian perspective. J Trop Pediatr. 2016;62:55-62.

10. De Klerk P, Van Dijk M, Van As A. Treatment and outcome of unusual animal bite injuries in young children. S Afr Med J. 2016;106:206-9.

11. Villagra V, Cáceres D, Alvarado S, Salinas E, Loreto M, Lucero E, et al. Características epidemiológicas de mordeduras en personas, según registro de atención de urgencias Provincia de Los Andes, Chile. Rev Chil Infectol. 2017;34:212-20.

12. Jofré L, Perret C, Abarca C, Solari V, Olivares R, López J, et al. Recomendaciones para el manejo de mordeduras ocasionadas por animales. Rev Chil Infectol. 2006;23:20-34.

13. Cedrés A, Morosini F, Margni C, López A, Alegretti M, Dall'Orso P, et al. Mordeduras por animales en niños. ¿Cuál es la situación actual en el Departamento de Emergencia Pediátrica del Centro Hospitalario Pereira Rossell? Arch Pediatr Urug. 2018;89:15-20.

14. Ogden R, Hedican E, Stach L, Herigon J, Jackson M, Newland J. Antibiotic management of animal bites in children during the methicillin-resistant Staphylococcus aureus era. J Pediatric Infect Dis Soc. 2013;2:379-81.

15. Liu-Wu Y, Orozco A. Tratamiento de las mordeduras de perro. Rev Med Costa Rica Centr Amer. 2014;71:289-92.

16. Piñeiro R, Carabaño I. Manejo práctico de mordeduras en atención primaria y en nuestro medio. Rev Pediatr Aten Primaria. 2015;17:263-70.

17. Sabhaney V, Goldman R. Management of dog bites in children. Can Fam Physician. 2012;58:1094-6.

18. Vázquez P, Guerrero MM. Mordeduras y picaduras. An Pediatr Contin. 2008;6:76-84

19. Oksi J, Laiho A, Laine M. Medical care after cat bites: a typical case and review. Int J Clin Med. 2014;5:340-3.

20. Suárez O, López JC, Burgos L, Aguilar R, Luis A, Encinas JL, et al Reconstrucción quirúrgica de las lesiones graves por mordedura de perro en niños. Cir Pediatr. 2017;20:148-50.

21. Velázquez V, Flores M, Gómez A. Tratamiento de heridas por mordedura de perro en región craneofacial. Rev Odont Mex. 2013;17:247-55.

22. Agrawal A, Kumar P, Singhal R, Singh V, Bhagol A. Animal bite injuries in children: review of literature and case series. Int $\mathrm{J}$ Clin Pediatr Dent. 2017; $10: 67-72$

23. Organización Panamericana de la Salud. Comisión Nacional de Zoonosis. Guía práctica de hidatidosis y zoonosis desatendidas. Disponible en: http://www.zoonosis.gub.uy/webzoonosis/materiales/pdf/guia_practica_ zoonosis.pdf

24. Baldovino R, Moreira N, Fernández A, Ferré A, Guerra M, Jaureguiberry J, et al. Loxoscelismo cutáneo. A propósito de un caso clínico. Arch Pediatr Urug. 2012;83:273-7.

25. Harz-Fresno I, Manterola P, Ruiz M, Abud C. Loxoscelismo cutáneo visceral: actualización en el manejo a propósito de un caso. Rev Chil Infectol. 2015;32:230-3.

26. López A, Ponce O. Mordedura de serpiente en niños. Rev Honduras Pediátrica. 1987;11:11-4.

27. Rodríguez S, Negrin A, Burger M. Efecto adverso por suero antibothrópico. Rev Med Urug. 2004;20:228-32.

28. Morais V. Análisis comparativos de los venenos ofídicos de importancia clínica y estudio bioquímico de los accidentes ofídicos en Uruguay. [Tesis de posgrado]. Montevideo: Facultad de Química, Universidad de la República; 2012

29. Tavares AV, Araújo KAM, Marques MRV, Vieira AA, Leite RS. The epidemiology of snakebite in the Rio Grande do Norte State, Northeastern Brazil. Rev Inst Med Trop Sao Paulo. 2017;59:e52. 